\title{
Engineering piezoresistivity using biaxially strained silicon
}

Pedersen, Jesper Goor; Richter, Jacob; Brandbyge, Mads; Thomsen, Erik Vilain; Hansen, Ole

Published in:

Applied Physics Letters

Link to article, DOI:

$10.1063 / 1.3059557$

Publication date:

2008

Document Version

Publisher's PDF, also known as Version of record

Link back to DTU Orbit

Citation (APA):

Pedersen, J. G., Richter, J., Brandbyge, M., Thomsen, E. V., \& Hansen, O. (2008). Engineering piezoresistivity using biaxially strained silicon. Applied Physics Letters, 93(26), 263501. https://doi.org/10.1063/1.3059557

\section{General rights}

Copyright and moral rights for the publications made accessible in the public portal are retained by the authors and/or other copyright owners and it is a condition of accessing publications that users recognise and abide by the legal requirements associated with these rights.

- Users may download and print one copy of any publication from the public portal for the purpose of private study or research.

- You may not further distribute the material or use it for any profit-making activity or commercial gain

- You may freely distribute the URL identifying the publication in the public portal

If you believe that this document breaches copyright please contact us providing details, and we will remove access to the work immediately and investigate your claim. 


\title{
Engineering piezoresistivity using biaxially strained silicon
}

\author{
Jesper Goor Pedersen, ${ }^{1, a)}$ Jacob Richter, ${ }^{2}$ Mads Brandbyge ${ }^{2}$ Erik V. Thomsen, ${ }^{2}$ \\ and Ole Hansen ${ }^{2,3}$ \\ ${ }^{1}$ Department of Photonics Engineering, Technical University of Denmark, DTU Fotonik, \\ DK-2800 Kongens Lyngby, Denmark \\ ${ }^{2}$ Department of Micro- and Nanotechnology, Technical University of Denmark, DTU Nanotech, \\ DK-2800 Kongens Lyngby, Denmark \\ ${ }^{3}$ Danish National Research Foundation's Center for Individual Nanoparticle Functionality (CINF), \\ Technical University of Denmark, DK-2800 Kongens Lyngby, Denmark
}

(Received 12 September 2008; accepted 9 December 2008; published online 29 December 2008)

\begin{abstract}
We calculate the shear piezocoefficient of $p$-type silicon with grown-in biaxial strain using a $6 \times 6 \mathbf{k} \cdot \mathbf{p}$ method. We find a significant increase in the value of the shear piezocoefficient for compressive grown-in biaxial strain, while tensile strain decreases the piezocoefficient. The dependence of the piezocoefficient on temperature and dopant density is altered qualitatively for strained silicon. In particular, we find that a vanishing temperature coefficient may result for silicon with grown-in biaxial tensile strain. These results suggest that strained silicon may be used to engineer the piezoresistivity to enhance the performance of piezoresistive stress sensors. (C) 2008 American Institute of Physics. [DOI: 10.1063/1.3059557]
\end{abstract}

By growing a layer of silicon on top of a $\mathrm{Si}_{x} \mathrm{Ge}_{1-x}$ substrate, the silicon layer, having a smaller lattice constant, must increase its in-plane lattice constant in order to match the underlying structure. In this way, a silicon layer with a tensile grown-in biaxial strain in the normal plane results. Similarly, a layer with a compressive biaxial strain in the normal plane may be fabricated by growing $\mathrm{Si}_{x} \mathrm{Ge}_{1-x}$ on top of an ordinary silicon substrate, as shown schematically in the insets of Fig. 1. Such strained silicon structures have rapidly become key players in the semiconductor industry due to the increased carrier mobility found in these structures. ${ }^{1}$ This increase in carrier mobility of strained silicon has been studied extensively, both theoretically and experimentally. ${ }^{2-7}$ Measured piezocoefficients of unstrained silicon surface channels have been used to explain sufficiently the transport in $n$ - and $p$-channel metal-oxide semiconductor devices in strained silicon. ${ }^{6,7}$ However, use of silicon with grown-in biaxial strain for piezoresistive sensing purposes, where higher accuracy is needed since piezoresistance is the primary effect, has not yet received much attention, and only few experimental results exist. ${ }^{8}$ In this paper we study theoretically the piezoresistivity of $p$-type silicon with grown-in biaxial strain by calculating the change in transport properties of the material in response to a small additional shear stress.

Biaxially strained (001) silicon has two independent shear piezocoefficients $\pi_{44}=\pi_{55} \neq \pi_{66}$ due to lower symmetry. ${ }^{8}$ Of these, $\pi_{66}$ is the technologically most important piezocoefficient in $p$-type silicon, and we consequently focus on $\pi_{66}$ in this paper. The in-plane strain in strained silicon structures is $\epsilon_{\|}=\epsilon_{1}=\epsilon_{2}$, while the growth direction strain is $\epsilon_{3}=-2 \epsilon_{\|} C_{12} / C_{11}$, determined using the condition of zero stress in the growth direction, $X_{3}=0$. Here $C_{i j}$ are the elements of the stiffness tensor of silicon. With this grown-in strain the resistivity $\varrho$ and conductivity $\boldsymbol{\sigma}$ tensors are diagonal with $\varrho_{1}=\varrho_{2} \neq \varrho_{3}$ and $\sigma_{1}=1 / \varrho_{1}$. Here, six vector notations are used for all tensor elements. When the material is

${ }^{a)}$ Electronic mail: jeped@fotonik.dtu.dk. subsequently stressed by an applied in-plane shear stress, $X_{6} \equiv X_{12}$, the conductivity and resistivity tensors are changed by the amount $\Delta \boldsymbol{\sigma}$ and $\Delta \varrho$ with the elements $\Delta \sigma_{6} \simeq$ $-\sigma_{1} \pi_{66} X_{6}$ and $\Delta \varrho_{6} \simeq \varrho_{1} \pi_{66} X_{6}$. We calculate the bandstructure of the prestrained and shear stressed material, and directly use that to calculate $\sigma_{1}$ and $\Delta \sigma_{6}$. The shear piezocoefficient is then calculated using $\pi_{66} \simeq-\Delta \sigma_{6} /\left(\sigma_{1} X_{6}\right)$. The approximations indicated above are related to the use of $\sigma_{1}$ instead of $\sqrt[3]{\sigma_{1}^{2} \sigma_{3}}$ for calculation of the relative change in conductivity. In the remainder of the paper, when we speak of strain, we are referring to the grown-in biaxial strain of the strained silicon structure rather than the strain due to the additional applied in-plane shear stress.

We base our band structure calculations on a $6 \times 6 \mathbf{k} \cdot \mathbf{p}$ model, ${ }^{9,10}$ while conductivities are calculated using the Boltzmann transport equation, including energy-dependent relaxation times. The model and the numerical methods used are explained in detail in a recent publication, ${ }^{11}$ where we find good agreement with experimental data for unstrained silicon. ${ }^{12}$ In the model scattering rates due to acoustic phonons, nonpolar optical phonons, and ionized impurities are included in order to accurately reproduce the doping density dependence of the shear piezocofficient. ${ }^{11}$ All calculations presented are based on pure silicon parameters, also in the case of compressive biaxial strain. However, we verified that using $\mathrm{Si}_{x} \mathrm{Ge}_{1-x}$ parameters ${ }^{13}$ in the case of compressive biaxial strain does not alter the results significantly and, in particular, the qualitative behavior remains unaffected. Strain, rather than material composition, is thus found to be the main cause of the observed changes in the shear piezocoefficient.

In Fig. 1 we show $\pi_{66}$ as a function of temperature and dopant density for the three cases of silicon and strained silicon with a grown-in biaxial strain of $\epsilon_{\|}=0.004$ (tensile) and $\epsilon_{\|}=-0.004$ (compressive), respectively. These values correspond to a Ge content of approximately $10 \%$. In the case of a compressive biaxial strain, the value of the piezocoefficient is significantly increased, and we also note a more pronounced temperature dependence. However, the overall 


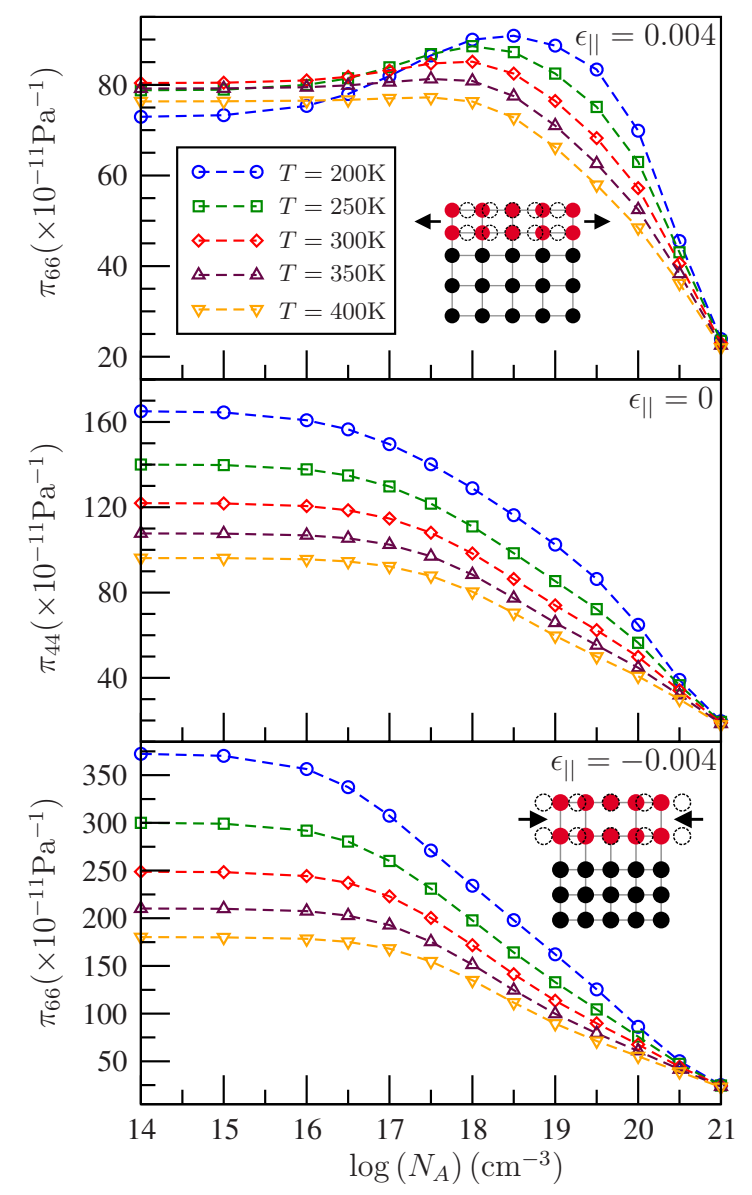

FIG. 1. (Color online) Shear piezocoefficient $\pi_{66}$ as a function of temperature and dopant density. Results are shown for silicon and for strained silicon with a grown-in biaxial strain of $\epsilon_{\|}=0.004$ (tensile) and $\epsilon_{\|}=-0.004$ (compressive), respectively. The panel in the middle shows the shear piezocoefficient for unstrained silicon where $\pi_{66}=\pi_{44}$. The insets illustrate the fabrication of strained silicon layers.

trends of the behavior of the piezocoefficient as temperature and dopant density are varied remain the same as for silicon. This is contrary to the case of a tensile biaxial strain, where there is a qualitative difference in the dependence of the piezocoefficient on temperature and dopant density. In particular, for low temperatures, the piezocoefficient increases with the dopant density, while at low dopant densities, the piezocoefficient is seen to increase with temperature. This behavior is in stark contrast with the ordinary behavior of the piezoresistive effect.

In Fig. 2 we show the dependence of the shear piezocoefficient on the biaxial strain. Results are shown for four different dopant densities at temperatures of $T=200 \mathrm{~K}$ and $T=300 \mathrm{~K}$. In all cases the shear piezocoefficient is increased for compressive biaxial strain, while a tensile strain decreases the value of the piezocoefficient. The relation between the value of the piezocoefficient and the applied biaxial strain is roughly linear for compressive strain, whereas it is highly nonlinear in the case of tensile strain. This results in a kink at zero biaxial strain in the relationship between the shear piezocoefficient and the biaxial strain. The dependence of $\pi_{66}$ on the biaxial strain is significantly more pronounced for low dopant densities. These results match the trends of the experimental data available in Ref. 8.

These trends may be explained by examining the effect of the biaxial strain on the band structure of silicon. We
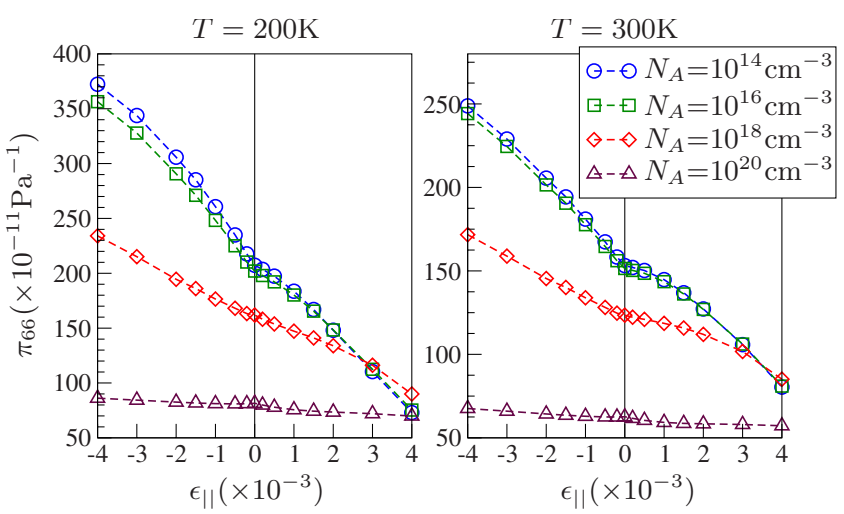

FIG. 2. (Color online) Piezocoefficient $\pi_{66}$ as a function of the magnitude of the grown-in biaxial strain for four different dopant densities. Results are shown for temperatures of $T=200 \mathrm{~K}$ and $T=300 \mathrm{~K}$, respectively. Note the different scales on the ordinates of the two figures.

illustrate this in Fig. 3 where we show the valence band structure of silicon along the [110] direction in the case of compressive and tensile biaxial strains, respectively. In both cases the heavy-hole $(\mathrm{HH})$ band remains relatively unaffected by the strain, while the light-hole (LH) and split-off (SO) bands are strongly affected. The coupling between the bands gives rise to avoided crossings in the band structure, which manifests itself as a pronounced warping of the bands and a strong divergence from the nearly parabolic nature of the unstrained band structure. In the case of tensile strain, the LH band is significantly increased in energy, while the SO band remains essentially unchanged. As a consequence the LH band becomes by far the dominant source of carriers. Due to the almost parabolic top of the LH band, the shear piezocoefficient is strongly reduced until the doping density is so high that the $\mathrm{HH}$ band also becomes populated. This explains why the reduction in shear piezocoefficient for tensile biaxial strain is significantly more pronounced for low dopant densities, while for $N_{A}=10^{20} \mathrm{~cm}^{-3}$ the piezocoefficient remains almost unaffected by the biaxial tensile strain. Also, as the dopant density is increased, the strongly warped band structure near the $\mathrm{HH}$ band is sampled, resulting in an increase in the shear piezocoefficient at low temperatures, as seen in Fig. 1. In the case of compressive biaxial strain, the opposite occurs, as the LH band is decreased in energy, while the SO band is increased correspondingly. The appearance of the $\mathrm{SO}$ band near the $\mathrm{HH}$ band results in a marked warping of the bands near the maximum of the valence band structure, giving rise to an increase in the shear piezocoefficient. However, the overall trends of the band structure in this case

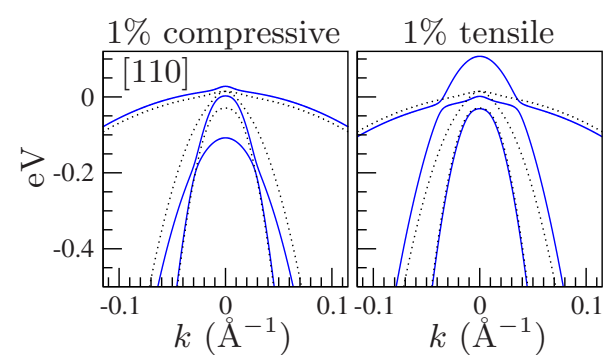

FIG. 3. (Color online) Band structures of strained silicon. The dotted black lines indicate the unstrained bands, while the solid blue lines are the bands of the strained material. Band structures are shown for compressive and tensile strains, respectively, and for the [110] direction in $\mathbf{k}$-space. The effect of biaxial strain is similar but less pronounced in other directions in $\mathbf{k}$-space. 

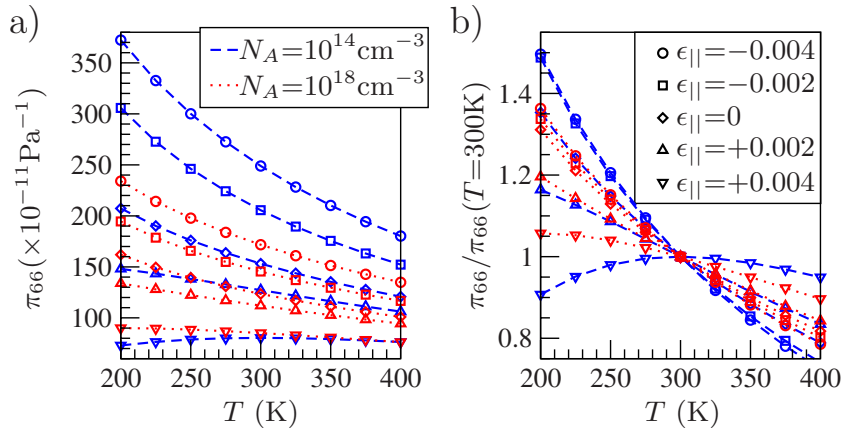

FIG. 4. (Color online) Piezocoefficient $\pi_{66}$ as a function of temperature for different magnitudes of the grown-in biaxial strain. Results are shown for two different dopant densities. Results in (b) are shown relative to the value of $\pi_{66}$ at $T=300 \mathrm{~K}$. Note that the legend is spread across both figures.

are very similar to the unstrained case, indicating why the behavior of the shear piezocoefficient for compressive biaxial strain is qualitatively the same as for ordinary silicon, as seen in Fig. 1.

In Fig. 4 we show the temperature dependence of the shear piezocoefficient for different magnitudes of the biaxial strain. We note that the increase in value of the piezocoefficient for compressive biaxial strains is accompanied by an increased temperature dependence. In Fig. 4(b) we also clearly see how in the case of tensile biaxial strain the temperature dependence decreases and eventually changes sign for low temperature and low doping as the magnitude of the strain is increased. This is interesting from a technological point of view as this means that it is possible by an optimal choice of doping and biaxial strain to adjust the temperature dependence, such that it is essentially zero for strained silicon structures. This may be particularly useful in situations where a decrease in the sensitivity of a device is acceptable if accompanied by a significant reduction in secondary dependencies.

In Fig. 5 we show the relative temperature dependence of $\pi_{66}$ as a function of the biaxial strain. We note that while the increase in $\pi_{66}$ for compressive biaxial strain is accompanied by a larger variation with temperature, the relative temperature dependence is not increased significantly, strengthening possible applications of such strained structures. Assuming that the temperature dependence of the shear piezocoefficient can be approximated as $\pi_{66}(T) \propto T^{\beta}$, we show in Fig. 5 the value of the temperature coefficient $\beta$ as a function of the magnitude of the biaxial strain. As a compressive biaxial strain is applied, the temperature dependence approaches the $1 / T$ dependence expected due to the inverse proportionality of the shear piezocoefficient and the kinetic energy of the carriers. This behavior is strongly modified with tensile biaxial strain for which the temperature coefficient increases, eventually becoming positive for low temperatures and dopant densities. A possible explanation of this behavior may be found in a delicate balance between two

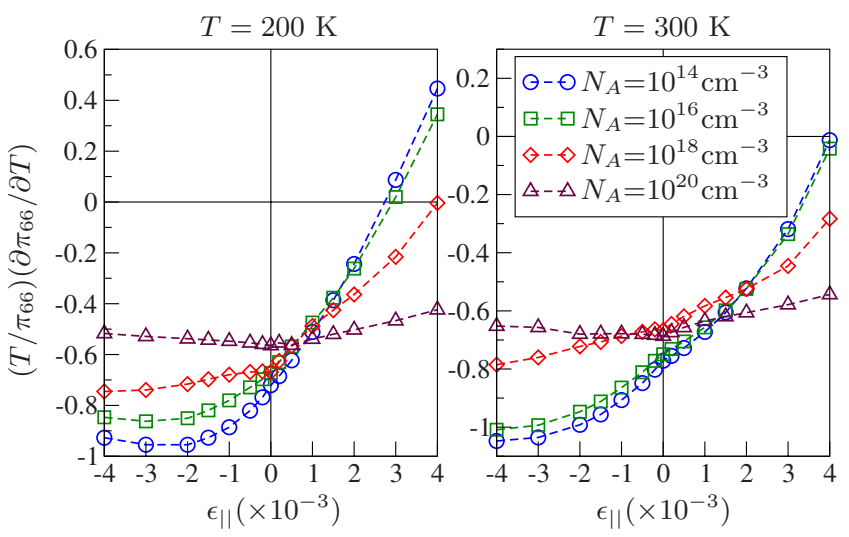

FIG. 5. (Color online) Temperature dependence of $\pi_{66}$ as a function of the magnitude of the grown-in biaxial strain for four different dopant densities. Results are shown for temperatures of $T=200 \mathrm{~K}$ and $T=300 \mathrm{~K}$, respectively. Note the different scales on the ordinates of the two figures.

opposing effects. First the piezoresistance effect in $p$-type material is inversely dependent on the average kinetic energy of the holes. Second large shear piezocoefficients only result when the strongly warped part of the $\mathrm{HH}$ band is sampled. With increasing temperature more and more carriers have sufficient energy to populate the $\mathrm{HH}$ band and add an increasing contribution to the piezocoefficient. In conclusion, we studied the effect of a grown-in biaxial strain on the shear piezocoefficient $\pi_{66}$ of silicon. Such a strain is achieved in strained silicon and strained $\mathrm{Si}_{x} \mathrm{Ge}_{1-x}$ structures. We find that $\pi_{66}$ is significantly altered due to the biaxial strain. In particular, the piezocoefficient can be increased nearly twofold in the case of compressive biaxial strain, while a tensile biaxial strain reduces the piezocoefficient. We studied the temperature dependence of $\pi_{66}$ and found that it can be strongly suppressed by introducing a tensile biaxial strain in silicon. These results suggest that strained silicon may be used to engineer the piezoresistivity to enhance the performance of piezoresistive stress sensors.

${ }^{1}$ F. Schäffler, Semicond. Sci. Technol. 12, 1515 (1997).

${ }^{2}$ E. X. Wang, P. Matagne, L. Shifren, B. Obradovic, R. Kotlyar, S. Cea, M. Stettler, and M. D. Giles, IEEE Trans. Electron Devices 53, 1840 (2006). ${ }^{3}$ E. Basaran, R. A. Kubiak, T. E. Whall, and E. H. C. Parker, Appl. Phys. Lett. 64, 3470 (1994).

${ }^{4}$ M. L. Lee and E. A. Fitzgerald, J. Appl. Phys. 94, 2590 (2003).

${ }^{5}$ T. Guillaume and M. Mouis, Solid-State Electron. 50, 701 (2006).

${ }^{6}$ S. E. Thompson, G. Sun, Y. S. Choi, and T. Nishida, IEEE Trans. Electron Devices 53, 1010 (2006).

${ }^{7}$ S. Suthram, J. C. Ziegert, T. Nishida, and S. E. Thompson, IEEE Electron Device Lett. 28, 58 (2007).

${ }^{8}$ J. Richter, O. Hansen, A. Nylandsted Larsen, J. Lundsgaard Hansen, G. F. Eriksen, and E. V. Thomsen, Sens. Actuators, A 123-124, 388 (2005).

${ }^{9}$ J. Hinckley and J. Singh, Phys. Rev. B 41, 2912 (1990).

${ }^{10}$ G. Dresselhaus, A. F. Kip, and C. Kittel, Phys. Rev. 98, 368 (1955).

${ }^{11}$ J. Richter, J. Pedersen, M. Brandbyge, E. V. Thomsen, and O. Hansen, J. Appl. Phys. 104, 023715 (2008).

${ }^{12}$ O. Tufte and E. Stelzer, J. Appl. Phys. 34, 313 (1963).

${ }^{13}$ Parameters for $\mathrm{Si}_{x} \mathrm{Ge}_{1-x}$ where approximated as simple linear combinations of the parameters of the individual constituents. 